\title{
UnA APROXIMACIÓn A LAS SOCIEDADES RURALES DE MÉXICO DESDE EL CONCEPTO DE APRENDIZAJE VICARIO
}

\author{
An Approach to Rural Societies in Mexico since the Concept of Vicarious Learning \\ Sergio Moctezuma-Pérez
}

Resumen: En este artículo se propone una reflexión sobre el concepto de aprendizaje vicario formulado por el psicólogo Albert Bandura y su aplicación en contextos no escolarizados. Se documentan los experimentos controlados que dieron origen al concepto, así como sus fundamentos teóricos y su aporte a los proyectos de cambio social. Se utilizan ejemplos de México y otras partes del mundo, y se describen las formas en las que el aprendizaje vicario se manifiesta en algunas sociedades rurales mexicanas. Se concluye que el aprendizaje vicario tiene potencial para generar estrategias que beneficien la educación no formal principalmente de jóvenes y adolescentes.

Palabras clave: Albert Bandura, aprendizaje vicario, aprendizaje activo, sociocognitivismo, sociedades rurales.

Abstract: This article considers the concept of vicarious learning as formulated by the psychologist Albert Bandura and as applied to learning in non-educational settings. It also documents the controlled experiments that gave rise to the concept, as well as to its theoretical foundations and their contribution to projects of social change. Examples from Mexico and other parts of the world are considered, placing special emphasis on how vicarious learning is manifested in certain rural areas of Mexico. In conclusion, the article discusses how the vicarious learning shows potential to generate educational strategies that would benefit youth and adolescents in non-formal settings.

Keywords: Albert Bandura, vicarious learning, activity learning, social cognitive theory, rural areas.

Sergio Moctezuma Pérez. Doctor en Antropología Social por la Universidad Iberoamericana, Ciudad de México. Profesor-investigador en el Instituto de Ciencias Agropecuarias y Rurales (ICAR) de la Universidad Autónoma del Estadode México. Temas deespecialización: antropología ecológica y la antropología de la alimentación. Correo electrónico: smoctezumap@uaemex.mx.
Enviado a dictamen: 7 de julio de 2016

Aprobación: 27 de abril de 2017.

Revisiones: 2. 


\section{Introducción}

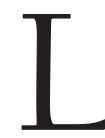
os hispanoparlantes suelen citar un refrán para justificar que un individuo debe vivir una situación para aprender sus consecuencias inherentes, a pesar de que éstas le hayan sido anticipadas por alguien más. Padres y docentes suelen utilizarlo para advertir a los niños y jóvenes acerca de las consecuencias de realizar actos negativos. Incluso, entre los adultos suele ser expresado continuamente cuando se ofrece un consejo que es desestimado. El refrán dice: "Nadie aprende en cabeza ajena". Sin embargo, el psicólogo canadiense Albert Bandura no estaría de acuerdo con las implicaciones pedagógicas que se encuentran detrás de este conocido refrán.

De acuerdo con Bandura (1977), los seres humanos podemos aprender las consecuencias de determinados actos mediante el proceso de experimentación. Sólo viviendouna situación específica oadoptandouna conducta determinada podemos establecer sus consecuencias. A lo anterior hay que añadir que los seres humanos también observamos los patrones de comportamiento de otros individuos y nos formamos un juicio sobre la pertinencia de apropiarnos de dichas conductas. Además de que aprendemos por experiencia propia, también incorporamos patrones que son aprendidos mediante la observación. El aprendizaje vicario, que implica la observación de comportamientos, se encuentra en el centro de interés de la teoría del aprendizaje social.

Una de las premisas más importantes para la teoría del aprendizaje social es la siguiente: las conductas que los individuos observan son aprendidas y reproducidas sólo en caso de representar un valor funcional, es decir, los comportamientos se evalúan a partir de su utilidad: si una conducta observada no representa una ventaja comparativa con otras o no tiene una aplicabilidad benéfica para el individuo, es muy probable que sea desechada. El aprendizaje vicario ocurre en cualquier momento de la vida, es decir, no se constriñe a una edad determinada ni está sujeto a los procesos de enseñanza formal (Bandura, 1977).

A partir de lo anterior, el objetivo del presente artículo es explicar la forma en que surge el concepto de aprendizaje vicario, formulado por el psicólogo Albert Bandura y se ofrece una explicación de las bases teóricas del concepto. El énfasis del documento se encuentra en la ejemplificación del concepto fuera del ámbito escolar —esto es, lejos del ámbito físico y pedagógico orientado hacia el proceso de enseñanza y aprendizaje - y se aplica a casos concretos de las sociedades urbanas y rurales del México contemporáneo.

\section{Aprendizaje vicario: experimentos controlados}

El psicólogo Albert Bandura desarrolló su teoría sobre el comportamiento humano en la Universidad de Stanford, en Estados Unidos, a mediados del siglo XX. Entre sus motivaciones profesionales se encontraba discutir con los psicólogos conductistas de la época acerca de las formas de aprendizaje de los individuos y el modelado de sus conductas. Lo anterior le permitió poner a discusión la idea de que el aprendizaje entre los seres humanos se lleva a cabo sólo por la experiencia directa de situaciones con consecuencias mensurables. Por el contrario, él centró su atención en la observación como fuente de aprendizaje (Bunge, 2008).

Para contextualizar los trabajos académicos de Bandura es necesario considerar que en la época en que comenzó sus investigaciones en Stanford, la televisión comenzaba a ganar terreno como medio de información y entretenimiento en los hogares estadounidenses. Desde ese entonces, él se preocupó por el contenido violento que era transmitido por la televisión y las repercusiones que tendrían las imágenes en las conductas de los infantes (Bunge, 2008). Dentro de la gran diversidad de experimentos que realizó Bandura, el del muñeco llamado Bobo es el más conocido entre psicólogos e interesados en las conductas sociales adquiridas por la observación e imitación.

El experimento se llevó a cabo a principios de 1960 y reunió a 36 niñas y 36 niños de entre tres y seis años de edad. De acuerdo con Bandura, Ross y Ross (1961), el experimento tuvo por objetivo observar el desarrollo de conductas agresivas en las niñas y niños. Él y sus 
colaboradores partían del supuesto de que si un niño observa modelos de conducta agresivos, reproducirá esa misma agresividad. Para corroborarlo, dividieron a los 72 niños en tres grupos: el primero fue expuesto a modelos agresivos, el segundo a modelos pasivos y un tercer grupo fue sólo de control.

Los niños participaron de manera individual, esto es, cada niño se encontraba en una habitación con la presencia de un adulto y con juguetes de diversos tipos: entre estos se encontraba el muñeco inflable llamado Bobo. En el caso de la exposición a conductas agresivas, el adulto después de unos minutos de divertirse calmadamente con los juguetes, se dirigía a Bobo y comenzaba a golpearlo, patearlo y mantenerlo en el suelo mientras se sentaba sobre él. A lo anterior se añadió violencia verbal contra Bobo. Después de jugar durante varios minutos de la forma descrita, el adulto abandonaba la habitación.

Por otro lado se encontraba el grupo de niños que no fue expuesto a ningún modelo de conducta agresiva. Entre ellos no surgió el interés por golpear e insultar a Bobo. De hecho, sólo las niñas y niños que observaron a los adultos golpear e insultar a Bobo manifestaron conductas agresivas una vez que el adulto abandonaba la habitación de juegos. Incluso, los niños fueron más agresivos que las niñas, y aún más si el adulto era hombre en lugar de mujer. Las niñas sólo mostraron mayor agresión física si el adulto era varón, y mayor agresión verbal si era mujer.

Con el experimento se comprobó que es posible incorporar conductas aprendidas por la observación. Incluso, Bandura, Ross y Ross (1963) publicaron los resultados de una variante del experimento de Bobo: expusieron a niños a modelos de conducta agresiva de manera presencial y a través de grabaciones proyectadas en televisión. Dicho experimento corroboró que los infantes son propensos a incorporar conductas agresivas/pasivas que observan en la vida real, así como la observada a través de los medios electrónicos. Tras su divulgación, las ideas de Bandura repercutieron profundamente en el medio académico y en diversos proyectos orientados al cambio social.

\section{Aprendizaje vicario: sus fundamentos teóricos}

En la primera sección del presente artículo se describieron dos experimentos que le permitieron a Bandura desarrollar su teoría. La practicidad de sus descubrimientos hizo posible que otros psicólogos realizaran investigaciones en torno a la teoría del aprendizaje social; sin embargo, el interés principal de este artículo es centrarse en los ejemplos prácticos y cotidianos sobre el aprendizaje vicario. Por lo anterior resulta necesario desarrollar con un poco de mayor profundidad los fundamentos teóricos del aprendizaje vicario. Hasta ahora se ha ejemplificado cómo surge y se utiliza este tipo de aprendizaje, pero el conocimiento de sus fundamentos teóricos permitirá revalorarlo.

Para Bandura (1977), el ser humano nace desprovisto de todo tipo de conductas que no sean biológicas. A medida que crece, el individuo observa conductas y emite juicios de valor en torno a las consecuencias —de aprobación y castigo- que también observa de ellas. El proceso que está implícito en el aprendizaje vicario puede explicarse por sus cuatro componentes. El primero de ellos es el proceso de atención, el cual implica que un individuo observe detenidamente algún acontecimiento relevante en su medio. Este proceso se acompaña de un rápido discernimiento sobre el valor funcional de lo que se está observando.

De acuerdo con Bandura, el medio en el que se desenvuelve un individuo y se relaciona con otros es fundamental para su modelamiento. De hecho, la gama de patrones de conducta se amplía enormemente a través de los medios. La televisión ejerce una importante influencia en los individuos de todas las edades, tal como lo demostró Bandura en 1963. Para el caso del México contemporáneo, además de la televisión se añaden como fuentes de modelado las diversas plataformas digitales — vía streaming — y las redes sociales más utilizadas Facebook, Twitter e Instagram- principalmente por los jóvenes, aunque sin excluir otros grupos de edad

El segundo proceso es el de la retención, el cual indica que, a pesar de que un individuo observe atentamente una conducta, ésta no será apropiada si es olvidada con facilidad. La información que un individuo recibe debe 
ser codificada y almacenada en su memoria, lo que se logra a través del uso de símbolos. Algunas conductas son retenidas por medio de un sistema de imágenes mentales, sobre todo cuando la actividad o conducta que se quiere imitar es difícil de describir mediante palabras, o bien cuando se ejecutan habilidades motoras que requieren de una repetición mecánica de procesos y decisiones.

Un segundo sistema de codificación está provisto por el lenguaje. Un individuo puede replicar conductas y actividades si es capaz de transformar la información en un listado de pasos a seguir. Por ejemplo, puede llevar a cabo procedimientos manuales si es capaz de recordar cuál paso es previo a otro. Ejemplo de lo anterior se encuentra en el aprendizaje de oficios como la albañilería, la carpintería, la herrería y demás, o en el caso de los agricultores que pretenden sembrar, cuidar, manejar y cosechar algún cultivo en específico. Es a través de las imágenes y de las palabras como los individuos repasan su aprendizaje.

El tercer proceso es el de la reproducción motora. Los dos procesos anteriores se encuentran en un nivel puramente cognitivo. En cambio, el tercer proceso involucra la conversión de las representaciones simbólicas en acciones específicas. Lo anterior significa pasar de lo observado a la práctica, y es en este punto donde el individuo se enfrenta a situaciones de ensayo y error. De acuerdo con Bandura, es difícil que un individuo no cometa errores al momento de poner en práctica lo que mentalmente ha aprendido, porque un individuo deberá perfeccionar o ajustar lo que ha observado con atención y codificado simbólicamente.

Por último, el cuarto proceso es el de la motivación: éste permite a un individuo seleccionar de aquello que ha aprendido lo que en verdad tiene interés en imitar, y también le permite desechar lo que no representa una ventaja o lo que es socialmente castigado. Es en este proceso donde se juzgan las consecuencias de los actos, ya sea porque el individuo los experimentó y fue capaz de objetivar las consecuencias de los actos o porque aprendió las consecuencias a través de la observación o el diálogo con otros individuos que experimentaron y aprendieron las consecuencias de determinados actos.
A partir de los cuatro procesos descritos, Bandura (1986) propuso que la conducta humana se explica por la interrelación de tres elementos: 1) los factores personales cognoscitivos, 2) las conductas y 3) el ambiente. A lo anterior Bandura lo llama modelo de reciprocidad triádica y explica cómo cada uno de los elementos condiciona a los otros dos. De esta forma, Bandura rompe con la tradición psicológica que pretendía explicar el comportamiento humano de manera unilineal, es decir, condicionadoúnicamente por los factores ambientales sin considerar la posibilidad de agencia. Para Bandura (1999), el ambiente puede clasificarse en: impuesto, seleccionado y construido.

La capacidad de adaptabilidad y resiliencia de los seres humanos tiene sus raíces en los sistemas sociales. Para Bandura (1999), los individuos son productores y al mismo tiempo producto de sus sistemas sociales. La relación de un individuo con su sociedad, así como sus factores cognitivos, condiciona su comportamiento porque el individuo nunca es pasivo ante las estructuras sociales y culturales que se encuentran en un ambiente. Creer lo contrario significaría que el individuo sólo es el producto de los factores supraindividuales y de procesos unidireccionales. En un mundo de este tipo no habría cabida para la diversidad social y cultural.

Actualmente los postulados de Bandura siguen vigentes para explicar la prevención de conductas de riesgo. Ejemplo de lo anterior es el trabajo de Palacios (2015), quien diseñó y aplicó un cuestionario entre 850 jóvenes estudiantes de la Ciudad de México. En dicho trabajo se presentaron seis conductas de riesgo - consumo de alcohol, tabaco, drogas, conducta sexual sin anticonceptivos, intento suicida y conducta antisocial- con las cuales las y los jóvenes se pueden identificar. El principal aporte fue el diseño y validación de un cuestionario que mide la capacidad de los jóvenes para aceptar o rechazar conductas, en este caso catalogadas como riesgosas.

Aunado a este trabajo, se encuentran aquellos que miden la autoeficacia ambiental (Palacios y Bustos, 2013), esto es, la capacidad que tienen los individuos -en este caso una muestra de trescientos jóvenes de la Ciudad de México - para conservar el ambiente, así 
como las implicaciones de esta capacidad para evaluar programas de educación ambiental. Los trabajos anteriores tienen como sustento el postulado de que la teoría debe fungir un papel importante en el cambio positivo de conductas mediante el diseño de mensajes efectivos utilizando la mercadotecnia social, así como el desarrollo de programas de intervención psicosocial (Palacios y Bustos, 2012).

\section{Aprendizaje vicario: proyectos de cambio social}

En México, los diversos trabajos de Bandura impactaron positivamente en los proyectos de cambio social iniciados por el reconocido poeta y dramaturgo mexicano Miguel Sabido Ruisánchez. Durante la década de 1970, Sabido se incorporó a Televisa para crear un modelo de televisión que él mismo denominó "entretenimiento con beneficio social". Sabido participó en la producción de varias telenovelas: Ven conmigo (1975), Acompáñame (1977), Vamosjuntos (1979), Caminemos (1980) y Nosotras las mujeres (1981) (Orozco, 2006). En estas producciones Sabido incorporaba personajes que generaban entre la audiencia una identificación positiva, en otros casos negativa, y personajes con conductas que cambian para bien (Guerrero, 2013).

En la telenovela Ven conmigo, Sabido utilizó para la trama el tema de la alfabetización entre los adultos, mientras que en Acompáñame fue el de la planificación familiar. En Vamos juntos se abordó la importancia de los valores dentro de la familia y de una buena autoestima, principalmente entre las mujeres. Con estas telenovelas, consideradas una trilogía, Sabido provocó cambios positivos dentro de la sociedad mexicana: después de abordar el tema de la alfabetización, el Instituto Nacional para la Educación de los Adultos (INEA) logró incrementar las cifras de adultos inscritos en los programas para aprender a leer y escribir.

En estos proyectos, junto a Televisa participaron la Secretaría de Educación Pública y la Secretaría de Saludque en esa época se denominaba Secretaría de Salubridad y Asistencia. Para ese entonces existía en los medios una postura acerca de las posibilidades benéficas de utilizar la televisión como un complemento de la educación que se brindaba al interior de las familias mexicanas y en las escuelas públicas. En 1997, la UNICEF patrocinó la producción de la séptima y última telenovela de Sabido: Los hijos de nadie, que abordó el tema de las niñas y niños en situación de calle y se orientó a crear consciencia sobre este problema (Singhal y Obregón, 1999).

El modelo de televisión creado por Sabido fue adoptado en países como Tanzania y la India. En el primero se realizó una radionovela llamada Twende na Wakati (Vamos con los tiempos) (1993-2002), que a lo largo de 676 episodios abordó diversos temas propios de la salud pública: planificación familiar, relaciones sexuales, prevención del VIH-Sida, entre otros. En la India se realizaron varias telenovelas y radionovelas con los mismos propósitos. Incluso, la Universidad Johns Hopkins retomó el trabajo de Miguel Sabido para crear campañas que promovieron la responsabilidad sexual entre adolescentes de América Latina, Filipinas y Nigeria (Singhal y Obregón, 1999).

También existen casos exitosos en Bolivia, Brasil, China y Pakistán (Smith, 2002) y, de acuerdo con Sabido (Singhal y Obregón, 1999), su modelo resultó pertinente por tres razones: 1) las telenovelas representan una mediación entre lo bueno y lo malo, es la audiencia quien decide sobre las conductas que ven en televisión; 2) el ser humano es propenso al chisme, lo que permite que los espectadores se interesen en las tramas, se mantengan pendientes de las conductas de los personajes y especulen acerca de lo que pasará en siguientes episodios, y 3) el ser humano siempre busca modelos de comportamiento que emular.

La televisión, de acuerdo con Sabido, no sólo ofrece la posibilidad de crear determinadas conductas de consumo entre los individuos, también puede promover conductas sociales positivas. De esta forma, Sabido fue capaz de aplicar el modelo sociocognitivo a sus proyectos creativos (Bandura, 2002). Él supo aprovechar que, durante las décadas de 1970 a 1990, el modelo televisivo en México apoyaba el entretenimiento con beneficio social. Al igual que los niños que formaron parte de los experimentos de Bandura, la televisión mexicana utilizó el aprendizaje vicario para que sus televidentes 
observaran conductas que deberían adoptar dado su potencial benéfico inmediato.

\section{Aprendizaje vicario: su utilización en el medio rural}

Las sociedades rurales e indígenas suelen utilizar el aprendizaje vicario para obtener y transmitir conocimientos y conductas en torno a las actividades agropecuarias. Lo anterior significa que el aprendizaje vicario está estrechamente relacionado con las diferentes formas de obtener el sustento en el medio rural. La observación e imitación como fuente de aprendizaje es una de las características que forman parte del conocimiento ecológico tradicional -o Traditional Ecological Knowledge (TEK) como se le conoce en lengua inglesa- Este tipo de conocimiento es diferente del científico que es producido y transmitido dentro de los márgenes de la ciencia occidental (Berkes, 1993).

De acuerdo con Taboada (2016), el proceso de transmisión cultural del conocimiento involucra cuatro elementos: 1) la información que debe transmitirse, 2) un modelo que posee la información, 3) un aprendiz que recibirá la información y 4) un contexto socioambiental donde ocurre el proceso. Para Taboada, así como para Bandura, el modelo puede ser un individuo o un personaje de un medio de comunicación — por ejemplo de la televisión-. En una sociedad rural, los aprendices observan el "prestigio social" que otros poseen por sus habilidades para cazar, pescar, sembrar, recolectar, curar o demás situaciones en las que son expertos.

En el caso de los habitantes de la isla Pukapuka — que forma parte de las Islas Cook de Nueva Zelanda-, infantes yadultos suelen aprender porobservaciónyposteriormente por imitación; son una sociedad principalmente visual. De hecho, las instrucciones son mínimas y se desalienta a los infantes a realizar cuestionamientos sobre cómo realizar algo. La segunda fuente de información consiste en escuchar las conversaciones entre personas. Deesta forma, observary repetir las conductas, así como escuchary poner en práctica lo escuchado, son los principales factores para transmitir el conocimiento entre los habitantes de la isla Pukapuka (Ruddle, 1993).
En el caso de las sociedades rurales de México, las niñas y niños aprenden las conductas que les serán necesarias en su etapa adulta por medio de la observación. En el ámbito rural, el aprendizaje vicario está estrechamente vinculado con las actividades que permiten obtener el sustento de las familias. De esta forma, a temprana edad las niñas observan a las mujeres de su grupo doméstico en un espacio muy específico: la cocina. En este lugar identifican cómo se prepara la masa de maíz para su transformación en tortillas, tamales o atoles, así como otros platillos propios de sus cocinas regionales.

En el caso de los niños, el aprendizaje se dirige a la consecución de actividades que forman parte del ciclo agrícola del maíz. Para lo anterior es necesario cómo utilizar los instrumentos de trabajo, las prácticas agrícolas que requieren los cultivos - abonos, manejo de plagas, control de la erosión del suelo, manejo de la infraestructura hidráulica, entre otros-, así como las formas en que se organizan los adultos — familiar y grupalmente- para realizar sus trabajos. Asimismo, el aprendizaje por observación y experimentación ha sido fundamental para el desarrollo paralelo de agriculturas similares en diversas partes del mundo (González, 2011).

Niñas y niños aprenden las nociones básicas de agricultura cuando acompañan a los adultos en el desgranado de las mazorcas. Esta actividad resulta completamente visual: es un proceso que el infante aprende teniendo a un adulto a su lado e imitando sus movimientos. De esta forma, adquiere el conocimiento suficiente como para realizar la actividad por sí solo, así como para enseñar en un futuro a los miembros más jóvenes de la unidad doméstica. Ejemplo de lo anterior se encuentra entre los totonacos de Mecatlán, en Veracruz, donde los infantes y jóvenes son los principales encargados de desgranar el maíz.

En repetidas ocasiones el autor de este artículo ayudó a desgranar el maíz y observó que los adultos participan sólo en contadas ocasiones. Esta actividad se realizaba por las tardes, al interior de la vivienda; mientras las mujeres hacían tortillas en la cocina o simplemente convivían entre ellas, los jóvenes colocaban en el suelo suficientes mazorcas como para 
media hora de trabajo. Además de los infantes y jóvenes que ya sabían desgranar, siempre se acercaba algún infante pequeño - un familiar o vecino, menor de cinco años - que sentado en el piso imitaba los movimientos que los demás hacíamos (Moctezuma, 2008).

Siguiendo el caso de la agricultura, los jóvenes y los adultos han incorporado nuevos cultivos en sus lugares de origen debido a la observación de las prácticas agrícolas que se realizan en otras partes del país o del extranjero. Cuando los individuos migran y se dedican a las labores agrícolas, utilizan una parte del conocimiento que ya poseen, al mismo tiempo que observan cómo personas con experiencia realizan actividades que ellos no conocían. De esta forma, los campesinos del sur del Estado de México aprendieron a cultivar fresas a partir de la observación vicaria en Estados Unidos (Juan, 2003).

En comunidades como Progreso Hidalgo, perteneciente al municipio de Villa Guerrero en el Estado de México, Juan (2014) ha documentado las diversas formas en que los campesinos adquieren y transmiten el conocimiento que poseen. Aunado a la transmisión oral, la vivencia y la experiencia individual como formas de aprendizaje, se encuentra la observación que da como resultado situaciones de ensayo y error. Las prácticas agrícolas que los campesinos observan son adoptadas y adaptadas al medio específico donde habitan los campesinos; aunque un campesino observe detalladamente una situación, existe la posibilidad de que no obtenga los resultados esperados al primer intento.

Lo anterior se corrobora con el estudio que realizó Reyes (2012) en la comunidad de Santiago Yeché perteneciente al Valle de Ixtlahuaca, Estado de México, donde los campesinos introdujeron el cultivo de tomate verde a finales de la década de 1980. Para el año 2002, de 31 familias entrevistadas el $64.5 \%$ se dedicaba a la siembra de tomate verde. Los campesinos reconocen expresamente que aprendieron observando a otros campesinos de la región y entre ellos mismos mediante la experimentación. Las ganancias económicas obtenidas del cultivo y la comercialización del tomate verde permitieron la adopción definitiva de una práctica aprendida por observación.
Existen otras actividades que se aprenden por observación: la alimentación de animales domésticos, partes específicas del proceso de preparación de alimentos y el cuidado de las plantas en los huertos familiares. Ejemplo de lo anterior se encuentra en San Francisco Tepeyanco, donde los niños y jóvenes observan cómo sus padres trasplantan diferentes especies vegetales al interior de un huerto. En segundo lugar, los adultos observan el cuidado y manejo de diversas especies y adoptan las prácticas si consideran que puede existir un mercado para comercializarlas, como en el caso de las plantas de ornato para las fiestas religiosas (Moctezuma, 2013).

Además de la agricultura, existen conductas aprendidas estrechamente vinculadas con la obtención del sustento mediante el trabajo asalariado. Por ejemplo, los mineros de Natividad, Oaxaca, aprendieron diversos trabajos que se realizan dentro y fuera de una mina por la simple observación (Murguía, 2016). De acuerdo con datos de las trayectorias laborales que la autora obtuvo de diversos extrabajadores, los encargados de la mina contrataban personal para realizar actividades manuales muy específicas. Estos individuos observaban las actividades que realizaban otros operadores y asimilaban los mecanismos implícitos. Esto les permitió ocupar las vacantes de esos puestos, previa demostración de que habían aprendido el trabajo.

La mina de Natividad se ubica en la sierra norte de Oaxaca, esto es, en una orografía accidentada. La capital del estado se encuentra a aproximadamente una hora y media en taxis colectivos y el poblamiento del municipio se dio por la instauración de la mina. Bajo este contexto fue necesario que los habitantes aprendieran oficios para trabajar dentro y fuera del socavón y, además, para proveer servicios a la localidad. Albañiles, herreros, soldadores y electricistas aprendieron mediante la observación a realizar sus oficios — principalmente de quienes trabajaban en la mina-, pero también por sus variadas experiencias migratorias (Murguía, 2016).

Con los ejemplos anteriores se puede comprender la importancia del aprendizaje vicario dentro del fenómeno de la migración. Esto lo ilustra con el conocido refrán "Al lugar que fueres, haz lo que vieres". 
En el caso de los migrantes mexicanos que por vez primera se desenvuelven en la sociedad estadounidense, aprenden normas y conductas de sus compatriotas o de otros individuos ajenos a esa sociedad, quienes les transmiten qué se debe y no hacer de manera verbal, pero también les indican que observen las conductas de los demás y traten de imitarlas: la finalidad es pasar desapercibido.

A lo anterior se añade el aprendizaje de conductas socialmente aprobadas que deben seguirse en eventos de carácter civil, religioso y ritual. Como se mencionó al inicio de este artículo, las conductas, además de ser observadas, son evaluadas y juzgadas a partir de su utilidad: sólo se imitan aquellas que traen consigo consecuencias positivas. Un ejemplo de patrones aprendidos que está fuera del ámbito agropecuario es una práctica que aún pervive en el medio rural de México -y de otras partes del mundo-y que varios investigadores han descrito: "el robo de la novia" (Goloubinoff, 2003; D'Aubeterre, 2003; Álvarez, 2007).

El noviazgo y el matrimonio en el México rural conllevan prácticas culturales aprendidas por observación, como en el robo de la novia. Esta es una práctica que vincula en parentesco a dos familias mediante el matrimonio de dos jóvenes. La conducta es la siguiente: un joven varón propone a su novia "fugarse" por un tiempo indeterminado a un lugar desconocido para sus familias. Posteriormente, el joven varón regresará a la comunidad para hablar con sus padres y con los de la novia. Después de ser reprendido por su conducta, ambas familias decidirán si aceptan el matrimonio de la pareja.

Aunque pueden existir casos en que las familias de los novios no aceptan que estos se casen, es una práctica socialmente aceptada. Los padres suelen reconocer que si sus hijos se quieren y se apoyan entre sí, no debería existir impedimento para llevar a cabo la celebración matrimonial. Por su parte, los jóvenes han observado cómo otros miembros de su sociedad han llevado a cabo dicha conducta y han conseguido su objetivo. Al mismo tiempo, ellos servirán como caso exitoso a emular por los jóvenes de su misma sociedad. Lo anterior fomenta que este patrón cultural sea adoptado y reproducido.

\section{Consideraciones finales}

Los aportes de Albert Bandura para la comprensión y explicación de la conducta humana nos permiten reflexionar sobre la influencia que tenemos como individuos en el actuar de otras personas y viceversa. Aunque sus nociones sobre los patrones de comportamiento se desarrollaron principalmente a mediados del siglo XX, su operatividad resulta completamente vigente. Los experimentos que realizó a principios de la década de 1960 son ilustrativos y clarificadores de procesos que acontecen en la actualidad. Por lo anterior, este psicólogo canadiense se ha ganado un lugar muy importante dentro de la psicología sociocognitiva y de la teoría del aprendizaje social.

Las consecuencias de los comportamientos que observamos son una fuente de información y motivación, y nuestra capacidad para juzgar una conducta y determinar el proceder en torno a ella permite iniciar un proceso cognitivo que en ocasiones es inconsciente. Sin embargo, como seres humanos somos proclives a tomar decisiones que originan consecuencias negativas, por lo que debiéramos aprovechar la infraestructura tecnológica para ofrecer entretenimiento educativo. Aunque existen programas de este tipo, se debe superar la idea de que la televisión comercial es contraria y excluyente de la educativa, es decir, retomar las ideas creativas que motivaron a Miguel Sabido en su profesión.

De la vida cotidiana tenemos ejemplos de cómo las consecuencias positivas fomentan en las personas el aprendizaje de conductas apreciadas, premiadas y reconocidas social y culturalmente. Además, éstas le permiten a los seres humanos adquirir información, conocimientos y habilidades que pueden aprovecharse como parte de las estrategias de sustento que se transforman en ganancias económicas. De esta forma, el aprendizaje vicario está estrechamente relacionado con la capacidad que tienen los individuos para adaptarse a un entorno ambiental, social, cultural, político y económico. Se parte de la idea de que el mundo es dinámico y los procesos de aprendizaje social también deben serlo. 
Por último, no basta con comprender cómo opera el aprendizaje vicario entre los seres humanos. Por el contrario, dado que se ha corroborado que este aprendizaje se puede dividir operacionalmente en cuatro procesos, es indispensable fomentar la generación de estrategias para beneficio de la educación formal y no formal. En los hogares es importante que los infantes y jóvenes tengan presentes modelos positivos y conductas apropiadas por seguir. En las escuelas se deben fomentar aprendizajes alternativos que sean respaldados por profesionales porque, de esta forma, se tienden puentes entre el hogar y la escuela como instancias socializadoras del adecuado desarrollo humano.

\section{Referencias bibliográficas}

Álvarez Arzate, María Dolores (2007). "Relaciones sociales y de parentesco en dos familias de Estelí, Nicaragua". En David Robichaux (coord.), Familia y diversidad en América Latina. Estudios de caso. Buenos Aires: CLACSO, pp. 355-373.

Bandura, A., D. Ross y S.A. Ross (1961). "Transmission of Aggression through Imitation of Aggressive Models". En Journal of Abnormal and Social Psychology, 63: 75-82.

Bandura, A., D. Ross y S.A. Ross (1963). "Imitation of Film-Mediated Aggressive Models". En Journal of Abnormal and Social Psychology, 63: 3-11.

Bandura, Albert (1977). Social Learning Theory. Nueva York: Prentice Hall.

Bandura, Albert (1986). Social Foundations of Thought and Action: A Social Cognitive Theory. New Jersey: PrenticeHalls.

Bandura, Albert (1999). "Social Cognitive Theory: An Agentic Perspective”. En Asian Journal of Social Psychology, 2: 21-41.

Bandura, Albert (2002). "Environmental Sustainability by Sociocognitive Deceleration of Population Growth". En P. Schmuch y W. Schultz (eds.). The Psychology of Sustainable Development. Nueva York: Kluwer, pp. 209-238. Berkes, Fikret (1993). "Traditional Ecological Knowledge in Perspective”. En Julian. T. Inglis (ed.), Traditional Ecological Knowledge: Concepts and Cases. Ottawa:
International Program on Traditional Ecological Knowledge, International Development Research Centre, pp. 1-9.

Bunge, Eduardo (2008). "Entrevista con Albert Bandura". En Revista Argentina de Clínica Psicológica, 12(2): 183-188.

D’Aubeterre, María Eugenia (2003). "Los múltiples significados de robarse la muchacha: el robo de la novia en un pueblo de migrantes del estado de Puebla”. En David Robichaux (comp.), El matrimonio en Mesoamérica ayer y hoy. México: Universidad Iberoamericana, pp. 249-264.

Goloubinoff, Marina (2003). "iPor qué se roba la novia? Las razones de una costumbre negada pero viva”. En David Robichaux (comp.), El matrimonio en Mesoamérica ayer y hoy. México: Universidad Iberoamericana, pp. 237-248.

González Jácome, Alba (2011). Historias varias, Un viaje en el tiempo con los agricultores mexicanos. México: Universidad Iberoamericana.

Guerrero Viguri, Raquel (2013). "De la telenovela a la webnovela. La fase experimental del formato en el modelo mexicano. Análisis histórico-estructural". Tesis de maestría en Estudios de la Cultura y la Comunicación, Universidad Veracruzana, México.

Juan Pérez, José Isabel (2003). "Tiempo con dinero y tiempo sin dinero. Agricultura tradicional y comercial en una zona de transición ecológica del Estado de México". Tesis de doctorado en Antropología Social, Universidad Iberoamericana, México.

Juan Pérez, José Isabel (2014). “Uso y manejo de recursos naturales en los procesos agrícolas de una comunidad del subtrópico mexicano. Progreso Hidalgo, México". En Revista Perspectivas Latinoamericanas, 11: 56-68. Nagoya, Japón: Universidad Nanzan.

Moctezuma Pérez, Sergio (2008). “Ambiente, cafeticultura y migración: los indígenas totonacos de Naranjales, Mecatlán, Veracruz". Tesis de maestría en Antropología Social, Universidad Iberoamericana, México.

Moctezuma Pérez, Sergio (2013). "San Francisco Tepeyanco: ambiente, cultura y agricultura". Tesis de doctorado en Antropología Social, Universidad Iberoamericana, México. 
Murguía Salas, María Verónica (2016). “'Hay que echarle ganas'. Estrategias de sustento de los extrabajadores mineros de Natividad, Oaxaca". Tesis de doctorado en Antropología Social, Universidad Iberoamericana, México.

Orozco Gómez, Guillermo (2006). "La telenovela en México: ¿de una expresión cultural a un simple producto para la mercadotecnia?" En Comunicación y Sociedad. 6, julio-diciembre: 11-35.

Palacios Delgado, Jorge (2015) "Estimación psicosométrica de la escala de autoeficacia ante conductas de riesgo para adolescentes en México". En Psychosocial Intervention, 24: 1-7.

Palacios Delgado, Jorge y José Marco Bustos (2013) "Validez factorial de la autoeficacia ambiental y su influencia estructural sobre la conducta proambiental en jóvenes". En Revista Iberoamericana de Evaluación en Psicología, 35(1): 95-111.

Palacios Delgado, Jorge y José Marco Bustos (2012) "La teoría como promotor para el desarrollo de intervenciones psicoambientales". En Psychosocial Intervention, 21(3): 245-257.
Reyes Montes, Laura (2012). Familia, agricultura y cultura en México. Un estudio antropológico. Alemania: Editorial Académica Española.

Ruddle, Kenneth (1993). "The Transmission of Traditional Ecological Knowledge". En Julian. T. Inglis (ed.), Traditional Ecological Knowledge: Concepts and Cases. Ottawa: International Program on Traditional Ecological Knowledge, International Development Research Centre, pp. 17-31.

Singhal, Arvind y Rafael Obregón (1999). "Social Uses of Commercial Soap Operas. A conversation with Miguel Sabido". En Journal of Development Communication. 10(1): 68-77.

Smith, Deborah (2002). "The Theory Heard around the World. Albert Bandura's Social Cognitive Theory is the Foundation of Television and Radio Shows that Have Changed the Lives of Millions". En Monitor on Psychology, 33: 30 .

Taboada Soldati, Gustavo (2016). "Local or Traditional Knowledge Transmission and Natural Resource Use". En U.P. Albuquerque y R. Alves (eds.), Introduction to Ethnobiology. Nueva York: Springer, pp. 235-238. 